\title{
LETTER
}

\section{The TSH-free thyroxine relationship: logarithmic-linear or error function?}

\author{
Melvin Khee-Shing Leow ${ }^{1,2,3}$ \\ ${ }^{1}$ Tan Tock Seng Hospital, Singapore 308433, Republic of Singapore, ${ }^{2}$ National University of Singapore, Singapore 117597, Republic of Singapore and \\ ${ }^{3}$ Brenner Center for Molecular Medicine, 30 Medical Drive, Singapore 117609, Republic of Singapore \\ (Correspondence should be addressed to M K-S Leow at Brenner Center for Molecular Medicine; Email: melvin_leow@sics.a-star.edu.sg)
}

In the paper by Hoermann et al. (1), a nonlinear model of the relationship between TSH and free thyroxine $\left(\mathrm{fT}_{4}\right)$ based on the error function is described. They dismissed the log-linear relationship in favor of the latter supported by sophisticated curve fitting and statistical software that produced a comparatively superior fit to their dataset.

Perhaps the modern age of biomedicine relies so heavily on advanced statistical techniques that few realize such methodologies used are not always appropriate to decipher the natural laws governing physiological processes. Depending on the interactions between the variables of interest, statistical regression may prove inadequate or even misleading. Within the realm of physiological sciences, most principles are conventionally deduced by direct observations on a single cell, tissue, or whole organism, and are replicated within and between experiments/research subjects with/without simulations rather than elucidating formulae from cross-sectional population database using computerized 'best-fit' procedures. The dynamic servomechanism of TSH- $\mathrm{fT}_{4}$ feedback obeys an inverse exponential power law, which is mathematically transformable to the log-linear function (2). Evidently, to capture the full essence of how this law operates in vivo, it is imperative to investigate serum TSH while subjecting a healthy animal through a wide spectrum of $\mathrm{fT}_{4}$ induced by thyroid hormones and antithyroid drugs. Thyroid cancer patients with total thyroidectomy on TSH suppression rendered hypothyroid by levothyroxine $\left(\mathrm{L}-\mathrm{T}_{4}\right)$ withdrawal prior to radioiodine uptake scanning, which represents an appropriate human model with which to explore and determine the relationship. In-depth studies of complete $\mathrm{TSH}-\mathrm{fT}_{4}$ dose-response curves for an adequately powered sample would be robust enough to derive the mathematical relationship governing the hypothalamus-pituitarythyroid (HPT) axis. A very recent study by Benhadi et al. (3) published in this journal (Feb 2010 issue) in which thyroid hormones were used to perturb the HPT axis continued to lend strong credence to the log-linear relationship. On the contrary, using empirical curvefitting algorithms on random, single time-point TSH-f $\mathrm{T}_{4}$ of disparate individuals with different thyroid function could potentially confuse rather than inform underlying physiological insights.
Hoermann et al. encountered data scatter such that three distinct segments of $\mathrm{fT}_{4}$ were needed for the loglinear plots, leading them to surmise that this relationship was inferior. Sources of significant data dispersion in a large population should be re-examined in the light of known HPT axis physiology. Apart from diurnal variations (4) and different interindividual HPT homeostatic set points (3), TSH secretion also exhibits clockwise hysteresis (2) with markedly different TSH, despite similar $\mathrm{fT}_{4}$ concentrations depending on thyroid functional states. In this context, because each data point represents a separate patient, even though the scatter-plot of TSH-f $\mathrm{T}_{4}$ coordinates appeared to be better fitted using an error function, it is erroneous to conclude that the error function formula (ERF) supersedes the log-linear model as their thyroid states were static and were not oscillated to unravel dose-response characteristics. Additionally, their error function revealed that TSH declined to 0 when $\mathrm{fT}_{4}$ exceeds $32 \mathrm{pmol} / \mathrm{l}$ or attained a peak of nearly $100 \mathrm{mIU} / \mathrm{l}$ when $\mathrm{fT}_{4}$ vanishes, a bias partly resulting from the working range of the TSH enzyme immunoassay of $0.01-100 \mathrm{mIU} / \mathrm{l}$. The loglinear model, however, does not assume that TSH declines to zero or rises to a maximum at those $\mathrm{fT}_{4}$ concentrations. If each patient's distinct $\log \mathrm{TSH}-\mathrm{fT}_{4}$ graph were generated and superimposed onto their plots, much of their data may in fact be found to substantiate the log-linear relationship, which to date remains a valid model for this aspect of HPT axis physiology.

\section{Declaration of interest}

The author declares that there is no conflict of interest that could be perceived as prejudicing the impartiality of the research reported.

\section{Funding}

This research did not receive any specific grant from any funding agency in the public, commercial, or not-for-profit sector.

\section{References}

1 Hoermann R, Eckl W, Hoermann C \& Larisch R. Complex relationship between free thyroxine and TSH in the regulation of thyroid function. European Journal of Endocrinology $2010 \mathbf{1 6 2}$ 1123-1129. (doi:10.1530/EJE-10-0106) 
2 Leow MK. A mathematical model of pituitary-thyroid interaction to provide an insight into the nature of the thyrotropin-thyroid hormone relationship. Journal of Theoretical Biology $2007 \mathbf{2 4 8}$ 275-287. (doi:10.1016/j.jtbi.2007.05.016)

3 Benhadi N, Fliers E, Visser TJ, Reitsma JB \& Wiersinga WM. Pilot study on the assessment of the setpoint of the hypothalamicpituitary-thyroid axis in healthy volunteers. European Journal of Endocrinology 2010162 323-329. (doi:10.1530/EJE-09-0655)
4 Chan V, Jones A, Liendo-Ch P, McNeilly A, Landon J \& Besser GM. The relationship between circadian variations in circulating thyrotropin, thyroid hormones and prolactin. Clinical Endocrinology 19789 337-349. (doi:10.1111/j.1365-2265.1978.tb02219.x)

Received 14 July 2010

Accepted 29 July 2010 